\title{
Joint spectral radius and ternary hermite subdivision
}

\author{
M. Charina ${ }^{1} \cdot$ C. Conti ${ }^{2}$ (D) $\cdot$ T. Mejstrik ${ }^{1}$ - J.-L. Merrien ${ }^{3}$
}

Received: 20 May 2020 / Accepted: 19 February 2021 / Published online: 23 March 2021

(C) The Author(s) 2021

\begin{abstract}
In this paper we construct a family of ternary interpolatory Hermite subdivision schemes of order 1 with small support and $\mathcal{H C}^{2}$-smoothness. Indeed, leaving the binary domain, it is possible to derive interpolatory Hermite subdivision schemes with higher regularity than the existing binary examples. The family of schemes we construct is a two-parameter family whose $\mathcal{H C}^{2}$-smoothness is guaranteed whenever the parameters are chosen from a certain polygonal region. The construction of this new family is inspired by the geometric insight into the ternary interpolatory scalar three-point subdivision scheme by Hassan and Dodgson. The smoothness of our new family of Hermite schemes is proven by means of joint spectral radius techniques.
\end{abstract}

Keywords Hermite subdivision · Hermite interpolation · Joint spectral radius · Taylor operator

Mathematics Subject Classification (2010) MSC 41A60 - MSC 65D15 - 13P05

Communicated by: Larry L. Schumaker

C. Conti

costanza.conti@unifi.it

M. Charina

maria.charina@univie.ac.at

T. Mejstrik

tommsch@gmx.at

J.-L. Merrien

jmerrien@insa-rennes.fr

1 Fakultät für Mathematik, Universität Wien, Oskar-Morgenstern-Platz 1, 1090, Wien, Austria

2 DIEF, Università di Firenze, Viale Morgagni 40/44, 50134, Firenze, Italy

3 Univ. Rennes, INSA Rennes, CNRS, IARMAR-UMR 6625, F-35000, Rennes, France 


\section{Introduction}

This paper focuses on Hermite subdivision schemes which are iterative algorithms for approximation or interpolation of given vector-valued discrete data consisting of function values and associated consecutive derivatives. Our main goal is to show, by applying the joint spectral radius techniques, that even in the Hermite case ternary subdivision schemes achieve higher smoothness than binary subdivision schemes. Furthermore we show that this can be done without a significant increase of the support of the mask. It is well known that such a phenomenon occurs in the scalar case, see e.g. [23]. However, the Hermite ternary case is more challenging.

\subsection{Motivation and novel contributions}

It is well known that it is a difficult task to keep the support size of a subdivision scheme small while increasing the smoothness of its limit functions. These two notions are mutually conflicting because high smoothness generally requires masks of large support. The large support however leads to the undesired more global influence of each initial data value on the limit function. Increasing the a-rity of the subdivision scheme is one of the possible ways to overcome this problem.

In this paper, we propose a new two-parameter family of ternary three-point Hermite schemes of order 1 for vector-valued data consisting of function values and associated first derivatives. The parameters are used to control the convergence and the regularity of the corresponding Hermite scheme. Indeed, the clever choice of these parameters guarantees that our Hermite schemes are $\mathcal{H C}^{2}$-convergent instead of $\mathcal{H C}^{1}$-convergent, which is the usual regularity expected for any scheme of order 1. In Section 2.2, in order to derive our family of Hermite schemes, we provide a new geometric interpretation of the family of ternary interpolatory scalar three-point subdivision schemes in [22]. The family in [22] is a one-parameter family which is $C^{1}$-smooth for a certain parameter range. In Section 2.3, we extend this scalar scheme to the Hermite case. The analysis of the convergence and regularity of the Hermite family is given in Section 3, where we combine the joint spectral radius approach for regularity analysis of the corresponding vector subdivision schemes with the $\mathcal{H C}^{0}$. convergence analysis of the Hermite schemes. The combination of these different methods, allows us to identify the convergence and regularity regions of the parameter plane and to identify the optimal parameter pairs in Section 4. In particular, our main result, Theorem 1 in Section 3, states that the family of Hermite schemes with the masks in (15) and parameters $(\lambda, \mu)$ from the polygonal domain $K_{2}$ in (21) possess the following properties

(i) the limit functions interpolate the data;

(ii) the limit functions are at least $C^{2}$-smooth;

(iii) the schemes are step-wise reproducing at least quadratic polynomials;

(iv) the masks are supported on $[-2,2]$.

Thus, we identify a whole class of Hermite interpolatory schemes whose regularity is higher than that of the existing binary schemes of the same order presented in [26]. 


\section{Construction of ternary hermite subdivision schemes}

In this section, we construct a two-parameter family of interpolatory ternary Hermite subdivision schemes of order $d=1$, i.e. of dimension 2. By construction, each scheme from this family reproduces quadratic polynomials. The suitable range of the parameters is given in Section 3. To derive this new family of Hermite scheme, we first give the necessary background from the subdivision theory, see Section 2.1 and then present, see Section 2.2, an alternative way for defining the ternary interpolatory scalar 3-point subdivision scheme in [22]. This new interpretation of the scheme is generalized to the Hermite case in Section 2.3.

\subsection{Background on vector and Hermite subdivision schemes}

Let $d \in \mathbb{N}_{0}$ be an integer. Vector subdivision schemes of dimension $d+1$ are iterative algorithms based on subdivision operators that generate denser and denser sequences $\boldsymbol{g}_{n}, n \in \mathbb{N}$, of vector-valued data from some initial vector-valued sequence $\boldsymbol{g}_{0}=$ $\left(\boldsymbol{g}_{0}(\alpha) \in \mathbb{R}^{d+1}, \alpha \in \mathbb{Z}\right)$ in $\ell^{d+1}(\mathbb{Z})$, the set of $d+1$-dimensional vector sequences indexed by $\mathbb{Z}$. Loosely speaking, if the ratio of the number of elements in $\boldsymbol{g}_{n+1}$ to the number of elements in $g_{n}$ is independent of $n$, then it is called the $a$-rity of the corresponding scheme.

In this paper we study ternary vector subdivision schemes. The associated leveldependent linear subdivision operators $S_{A_{n}}: \ell^{d+1}(\mathbb{Z}) \rightarrow \ell^{d+1}(\mathbb{Z})$, for any $n \in \mathbb{N}_{0}$, map the sequences $\boldsymbol{g}_{n}=\left(\boldsymbol{g}_{n}(\alpha), \alpha \in \mathbb{Z}\right)$ into $\boldsymbol{g}_{n+1}=\left(\boldsymbol{g}_{n+1}(\alpha), \alpha \in \mathbb{Z}\right)$ and are defined by

$$
\begin{gathered}
\boldsymbol{g}_{n+1}(\alpha):=\left(S_{\boldsymbol{A}_{n}} \boldsymbol{g}_{n}\right)(\alpha) \quad \alpha \in \mathbb{Z}, \quad n \in \mathbb{N}_{0}, \\
\left(S_{\boldsymbol{A}_{n}} \boldsymbol{g}_{n}\right)(\alpha):=\sum_{\beta \in \mathbb{Z}} \boldsymbol{A}_{n}(\alpha-3 \beta) \boldsymbol{g}_{n}(\beta), \quad \alpha \in \mathbb{Z}, \quad n \in \mathbb{N}_{0} .
\end{gathered}
$$

At each level $n$ of the subdivision recursion, the matrix coefficients in (2) are taken from the matrix-valued sequence $\boldsymbol{A}_{n}:=\left(\boldsymbol{A}_{n}(\alpha) \in \mathbb{R}^{(d+1) \times(d+1)}, \alpha \in \mathbb{Z}\right)$, called the level $n$ subdivision mask. In our case, the sequence $\left(\boldsymbol{A}_{n}, n \in \mathbb{N}_{0}\right)$ contains masks $\boldsymbol{A}_{n}$ of the same finite support, i.e. $\operatorname{supp} \boldsymbol{A}_{n}:=\left\{\alpha \in \mathbb{Z}: \boldsymbol{A}_{n}(\alpha) \neq \mathbf{0}\right\} \subseteq[-N, N]$ for some $N \in \mathbb{N}$. The $(d+1) \times(d+1)$ matrix-valued Laurent polynomials

$$
\boldsymbol{A}_{n}^{*}(z):=\sum_{\alpha \in \mathbb{Z}} \boldsymbol{A}_{n} z^{\alpha}, \quad z \in \mathbb{C} \backslash(0), \quad n \in \mathbb{N}_{0},
$$

are the associated mask symbols.

The vector subdivision scheme is the repeated application of the subdivision operators in (2) to the starting vector-valued sequence $\boldsymbol{g}_{0} \in \ell^{d+1}(\mathbb{Z})$, i.e.

$$
\left\{\begin{array}{l}
\text { Input } \quad\left(\boldsymbol{A}_{n}, n \in \mathbb{N}_{0}\right) \text { and } \boldsymbol{g}_{0} \\
\text { For } \quad n=0,1, \ldots \\
\\
\boldsymbol{g}_{n+1}:=S_{\boldsymbol{A}_{n}} \boldsymbol{g}_{n}
\end{array}\right.
$$

For the sake of simplicity it will be denoted as $\left(S_{A_{n}}, n \in \mathbb{N}_{0}\right)$.

This paper deals with two kinds of vector schemes of dimension $d+1$. The first one is a stationary vector subdivision scheme $S_{A}$ with the level-independent masks 
$\boldsymbol{A}_{n}=\boldsymbol{A}=\left(\boldsymbol{A}(\alpha) \in \mathbb{R}^{(d+1) \times(d+1)}, \alpha \in \mathbb{Z}\right), n \in \mathbb{N}_{0}$, and with the level-independent subdivision operators $S_{A_{n}}=S_{A}, n \in \mathbb{N}_{0}$. For simplicity, we call this stationary subdivision scheme $S_{A}$ as it is fully determined by the unique corresponding subdivision operator $S_{A}$. Such vector subdivision schemes have been studied by many authors e.g. $[1-3,5,7,12,25]$ and reference therein.

We continue by defining the convergence and the regularity of a level-dependent subdivision scheme $\left(S_{A_{n}}, n \in \mathbb{N}_{0}\right)$.

Definition 1 A ternary vector subdivision scheme $\left(S_{\boldsymbol{A}_{n}}, n \in \mathbb{N}_{0}\right)$ is called

i) convergent, if for every initial vector sequence $\boldsymbol{g}_{0} \in \ell^{d+1}(\mathbb{Z})$ and the corresponding sequence of refinements in (2), $\boldsymbol{g}_{n}=S_{\boldsymbol{A}_{n}} \boldsymbol{g}_{n-1}, n \in \mathbb{N}_{0}$, there exists a continuous vector function $\boldsymbol{\Phi}_{\boldsymbol{g}_{0}}: \mathbb{R} \rightarrow \mathbb{R}^{d+1}$, such that for every compact subset $K \subset \mathbb{R}$

$$
\lim _{n \rightarrow \infty} \max _{\alpha \in \mathbb{Z} \cap 3^{n} K}\left\|\boldsymbol{\Phi}_{g_{0}}\left(3^{-n} \alpha\right)-\boldsymbol{g}_{n}(\alpha)\right\|_{\infty}=0
$$

ii) $\mathcal{C}^{\ell}$-convergent, $\ell \in \mathbb{N}$, if $\boldsymbol{\Phi}_{g_{0}} \in \mathcal{C}^{\ell}(\mathbb{R})$ for every initial vector sequence $\boldsymbol{g}_{0}$ in $\ell_{\infty}^{d+1}(\mathbb{Z})$

iii) contractive, if $\boldsymbol{\Phi}_{\boldsymbol{g}_{0}}=\mathbf{0}$ for every initial sequence $\boldsymbol{g}_{0}$ in $\ell^{d+1}(\mathbb{Z})$.

The second type of vector subdivision schemes that we consider is Hermite subdivision schemes of dimension $d+1$ and order $d$. For a given mask $\boldsymbol{A}=$ $\left(\boldsymbol{A}(\alpha) \in \mathbb{R}^{(d+1) \times(d+1)}, \alpha \in \mathbb{Z}\right)$ with finite support, the associated linear Hermite subdivision scheme is based on special type of level-dependent (non-stationary) operators $S_{A_{n}}: \ell^{d+1}(\mathbb{Z}) \rightarrow \ell^{d+1}(\mathbb{Z})$ given by

$$
\boldsymbol{f}_{n+1}(\alpha):=\left(S_{\boldsymbol{A}_{n}} \boldsymbol{f}_{n}\right)(\alpha)=\sum_{\beta \in \mathbb{Z}} \boldsymbol{D}^{-n-1} \boldsymbol{A}(\alpha-3 \beta) \boldsymbol{D}^{n} \boldsymbol{f}_{n}(\beta), \quad \alpha \in \mathbb{Z}, \quad n \in \mathbb{N}_{0},
$$

with the diagonal matrix $\boldsymbol{D}=\operatorname{diag}\left(1,1 / 3, \ldots, 1 / 3^{d}\right) \in \mathbb{R}^{(d+1) \times(d+1)}$.

Note that the masks

$$
\boldsymbol{D}^{-n-1} \boldsymbol{A} \boldsymbol{D}^{n}=\left(\boldsymbol{D}^{-n-1} \boldsymbol{A}(\alpha) \boldsymbol{D}^{n}, \alpha \in \mathbb{Z}\right), \quad n \in \mathbb{N}_{0},
$$

in (4) have a very special type of level-dependence. For shortness, with a slight abuse of notation, we denote the Hermite subdivision scheme $\left(S_{D^{-n-1} \boldsymbol{A} \boldsymbol{D}^{n}}, n \in \mathbb{N}_{0}\right)$ simply with $H_{\boldsymbol{A}}$ to emphasize this special type of the dependency on the mask $\boldsymbol{A}$. Moreover, if $H_{A}$ is convergent, in the sense of Definition 2 given below, the vector-valued elements $f_{n}(\alpha)$ in (4) are to be interpreted, for large $n$, as approximations to the function values and the successive derivatives of the corresponding limit function $\Phi_{\boldsymbol{f}_{0}}=\lim _{n \rightarrow \infty} S_{\boldsymbol{D}^{-n-1} \boldsymbol{A} \boldsymbol{D}^{n}} \cdots S_{\boldsymbol{D}^{-1} \boldsymbol{A}} \boldsymbol{f}_{0}$ evaluated at $3^{-n} \alpha$, i.e.

$$
\boldsymbol{f}_{n}(\alpha)=\left(\begin{array}{c}
f_{n, 1}(\alpha) \\
f_{n, 2}(\alpha) \\
\vdots \\
f_{n, d+1}(\alpha)
\end{array}\right) \approx \Phi_{f_{0}}\left(3^{-n} \alpha\right)=\left(\begin{array}{c}
\phi\left(3^{-n} \alpha\right) \\
\phi^{\prime}\left(3^{-n} \alpha\right) \\
\vdots \\
\phi^{(d)}\left(3^{-n} \alpha\right)
\end{array}\right), \quad \alpha \in \mathbb{Z}
$$


where $f_{n, j}(\alpha)$ denotes the $j$ th component of the vector $\boldsymbol{f}_{n}(\alpha)$.

Hermite schemes were introduced in [26] and have been studied by several authors from different perspectives e.g [9-11, 15, 17, 18, 21, 24].

We observe that, since (4) can be rewritten as

$$
\boldsymbol{D}^{n+1} \boldsymbol{f}_{n+1}(\alpha)=\sum_{\beta \in \mathbb{Z}} \boldsymbol{A}(\alpha-3 \beta) \boldsymbol{D}^{n} \boldsymbol{f}_{n}(\beta), \quad \alpha \in \mathbb{Z}, \quad n \in \mathbb{N}_{0},
$$

each Hermite subdivision operator $S_{D^{-n-1} A D^{n}}$ of order $d$ and the stationary vector operator $S_{A}$ of dimension $d+1$ are related by

$$
\boldsymbol{D}^{n+1}\left(S_{\boldsymbol{D}^{-n-1} \boldsymbol{A}_{n} \boldsymbol{D}^{n}} \boldsymbol{f}_{n}\right)(\alpha)=\left(S_{\boldsymbol{A}} \boldsymbol{D}^{n} \boldsymbol{f}_{n}\right)(\alpha), \quad \alpha \in \mathbb{Z}, \quad n \in \mathbb{N}_{0} .
$$

Thus, the (stationary) vector subdivision scheme $S_{A}$ is called the stationary counterpart of $H_{A}$.

We make use of the following notion of convergence of a Hermite subdivision scheme $H_{A}$ that better captures the intrinsic structure of Hermite subdivision schemes.

Definition 2 A ternary Hermite scheme $H_{\boldsymbol{A}}=\left(S_{D^{-n-1} \boldsymbol{A} D^{n}}, n \in \mathbb{N}_{0}\right)$ of order $d$, is called $\mathcal{H C}^{\ell}$-convergent with $\ell \geq d$, if for any initial vector sequence $\boldsymbol{f}_{0} \in \ell^{d+1}(\mathbb{Z})$ and the corresponding sequence of refinements $\boldsymbol{f}_{n}=S_{\boldsymbol{D}^{-n-1} \boldsymbol{A} \boldsymbol{D}^{n}} \cdots S_{\boldsymbol{D}^{-1} \boldsymbol{A}} \boldsymbol{f}_{0}, n \in$ $\mathbb{N}_{0}$, in (4), there exists a vector-valued function $\boldsymbol{\Phi}_{f_{0}}: \mathbb{R} \rightarrow \mathbb{R}^{d+1}$,

$\boldsymbol{\Phi}_{f_{0}}=\left[\phi^{(i)}\right]_{i=0, \ldots, d} \in C^{\ell-d}(\mathbb{R})$ with $\phi=\phi^{(0)} \in C^{\ell}(\mathbb{R})$ and $\phi^{(i)}=\frac{d^{i} \phi^{(0)}}{d x^{i}}, i=$ $1, \ldots, d$, such that for every compact subset $K \subset \mathbb{R}$

$$
\lim _{n \rightarrow \infty} \max _{\alpha \in \mathbb{Z} \cap 3^{n} K}\left\|\boldsymbol{\Phi}_{f_{0}}\left(3^{-n} \alpha\right)-\boldsymbol{f}_{n}(\alpha)\right\|_{\infty}=0 .
$$

The usually used notions of $C^{\ell}$-convergence for the vector scheme $\left(S_{A_{n}}, n \in \mathbb{N}_{0}\right)$ and $\mathcal{H C}^{\ell}$-convergence for the Hermite scheme $H_{A}$ are intrinsically different. The first notion in Definition 1 refers to the minimal smoothness of the entries in $\boldsymbol{\Phi}_{\boldsymbol{g}_{0}}$ while the second one in Definition 2 to the maximal one of the entries in $\boldsymbol{\Phi}_{f_{0}}$. In other words, due to (6), if we look at a $\mathcal{H C}^{\ell}$-convergent Hermite scheme simply as a

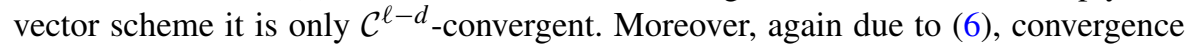
and regularity of $S_{A}$ does not imply convergence and regularity of $H_{A}$.

In this paper we make use of the following concept of ternary interpolatory subdivision scheme.

Definition 3 A ternary Hermite subdivision scheme $H_{A}$ is called interpolatory, if

$$
\boldsymbol{A}(0)=\boldsymbol{D} \quad \text { and } \quad \boldsymbol{A}(3 \alpha)=\mathbf{0}, \quad \alpha \in \mathbb{Z}, \quad \alpha \neq 0 .
$$

Note that a Hermite interpolatory scheme in (4) generates vector sequences satisfying

$$
\boldsymbol{f}_{n+1}(3 \alpha)=\boldsymbol{f}_{n}(\alpha), \quad \text { for } \quad \alpha \in \mathbb{Z}, \quad n \in \mathbb{N}_{0} .
$$




\subsection{Scalar case}

\subsubsection{Lagrange interpolants}

We start by determining two special cubic interpolants $P_{\ell}$ and $P_{r}$ to the data $\left(\alpha, p_{\alpha}\right)$ with $p_{\alpha} \in \mathbb{R}, \alpha \in\{-1,0,1\}$. The corresponding interpolation problems are underdetermined and we choose

$$
\begin{aligned}
& P_{\ell}(t):=p_{0}+\ell_{1} t+\ell_{2} t^{2}+\lambda\left(p_{-1}-2 p_{0}+p_{1}\right) t^{3}, \quad t \in \mathbb{R}, \\
& P_{r}(t):=p_{0}+r_{1} t+r_{2} t^{2}-\lambda\left(p_{-1}-2 p_{0}+p_{1}\right) t^{3}, \quad t \in \mathbb{R},
\end{aligned}
$$

to ensure $P_{\ell}(0)=P_{r}(0)=p_{0}$ and to introduce a free parameter $\lambda \in \mathbb{R}$. Note that $\lambda$ controls the second difference $p_{-1}-2 p_{0}+p_{1}$ and will be useful for controlling the regularity of the scalar ternary scheme in Section 2.2.2. The coefficients

$$
\begin{aligned}
& \ell_{1}=-\lambda\left(p_{-1}-2 p_{0}+p_{1}\right)+\frac{1}{2}\left(p_{1}-p_{-1}\right), \quad \ell_{2}=\frac{1}{2}\left(p_{-1}-2 p_{0}+p_{1}\right), \\
& r_{1}=\lambda\left(p_{-1}-2 p_{0}+p_{1}\right)+\frac{1}{2}\left(p_{1}-p_{-1}\right), \quad r_{2}=\frac{1}{2}\left(p_{-1}-2 p_{0}+p_{1}\right),
\end{aligned}
$$

are determined from two linear systems of equations derived from the interpolation conditions $P_{\ell}(\alpha)=P_{r}(\alpha)=p_{\alpha}$ for $\alpha \in\{-1,1\}$.

Remark 1 If $\pi \in \mathbb{P}_{1}$ and $p_{\alpha}=\pi(\alpha), \alpha \in\{-1,0,1\}$, then, by construction,

$$
P_{\ell}=P_{r}=\pi \text {. }
$$

Moreover, for $\lambda=0$, (9) holds for $\pi \in \mathbb{P}_{2}$, see Fig. 1 .

\subsubsection{Ternary scalar subdivision scheme}

To define the corresponding ternary scalar subdivision operator $S_{a}: \ell(\mathbb{Z}) \rightarrow \ell(\mathbb{Z})$,

$$
f_{n+1}:=S_{a} f_{n}=\sum_{\beta \in \mathbb{Z}} a(\cdot-3 \beta) f_{n}(\beta), \quad f_{n}=\left(f_{n}(\alpha), \alpha \in \mathbb{Z}\right), \quad n \in \mathbb{N}_{0},
$$

we choose the initial sequence $f_{0}=\left(f_{0}(\alpha), \alpha \in \mathbb{Z}\right)$ with

$$
f_{0}(\alpha)=p_{\alpha}, \quad \alpha \in\{-1,0,1\} \quad \text { and } \quad f_{0}(\alpha)=0, \quad \alpha \notin\{-1,0,1\},
$$
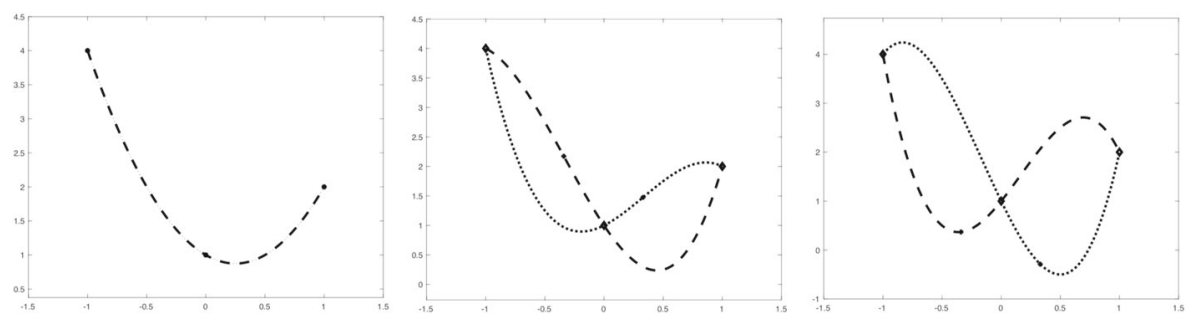

Fig. 1 Interpolants $P_{\ell}$ (dashed) and $P_{r}$ (dotted) for $\lambda=0,0.5,-1$, respectively, with the corresponding evaluation points 
and set

$$
f_{1}(-1)=P_{\ell}(-1 / 3), \quad f_{1}(0)=P_{r}(0)=P_{\ell}(0)=p_{0}, \quad f_{1}(1)=P_{r}(1 / 3) .
$$

Therefore, the following linear system of equations

$$
\begin{gathered}
\sum_{\beta \in\{-1,0,1\}} a(-1-3 \beta) p_{\beta}=P_{\ell}(-1 / 3)=\frac{1}{27}\left((6+8 \lambda) p_{-1}+(24-16 \lambda) p_{0}+(-3+8 \lambda) p_{1}\right), \\
\sum_{\beta \in\{-1,0,1\}} a(-3 \beta) p_{\beta}=p_{0}, \\
\sum_{\beta \in\{-1,0,1\}} a(1-3 \beta) p_{\beta}=P_{r}(1 / 3)=\frac{1}{27}\left((-3+8 \lambda) p_{-1}+(24-16 \lambda) p_{0}+(6+8 \lambda) p_{1}\right)
\end{gathered}
$$

uniquely identifies the mask

$$
a=(\ldots, 0, u, 0, v, 1-u-v, 1,1-u-v, v, 0, u, 0, \ldots),
$$

where $u:=\frac{-3+8 \lambda}{27}, v:=\frac{6+8 \lambda}{27}$ and where 1 is at the position $\alpha=0$. For every $\lambda \in \mathbb{R}$, the mask $a$ is symmetric and is supported on $[-4,4]$.

Note that $u=v-1 / 3$ and that (10) defines the one-parameter family of subdivision schemes in [22] whose convergence and $C^{1}$ smoothness is proved for $2 / 9<v<3 / 9$.

\subsection{A family of Hermite subdivision schemes of order 1}

\subsubsection{Hermite interpolants}

We generalize the idea described in Section 2.2 to the Hermite case. We start by solving the Hermite interpolation problem for the given data $\left(\alpha, p_{\alpha}, p_{\alpha}^{\prime}\right), \alpha \in\{-1,0,1\}$, and for two sextic Hermite interpolants of the form

$$
\begin{aligned}
& P_{H, \ell}(t):=p_{0}+p_{0}^{\prime} t+\sum_{i=1}^{4} \ell_{i} t^{i+1}+\left(\lambda \delta^{\prime}+\mu \delta\right) t^{6}, \\
& P_{H, r}(t):=p_{0}+p_{0}^{\prime} t+\sum_{i=1}^{4} r_{i} t^{i+1}-\left(\lambda \delta^{\prime}+\mu \delta\right) t^{6}
\end{aligned}
$$

with

$$
\delta^{\prime}=p_{-1}^{\prime}-2 p_{0}^{\prime}+p_{1}^{\prime} \quad \text { and } \quad \delta=\frac{p_{1}-p_{-1}}{2}-p_{0}^{\prime} .
$$

Note that the polynomials in (11) automatically satisfy the Hermite-type interpolation conditions

$$
P_{H, \ell}(0)=P_{H, r}(0)=p_{0} \quad \text { and } \quad P_{H, \ell}^{\prime}(0)=P_{H, r}^{\prime}(0)=p_{0}^{\prime} .
$$

Note also that the parameters $\lambda, \mu \in \mathbb{R}$ in (11) are introduced to control the differences $p_{-1}^{\prime}-2 p_{0}^{\prime}+p_{1}^{\prime}$ and $\frac{p_{1}-p_{-1}}{2}-p_{0}^{\prime}$ and influence the regularity of the corresponding Hermite subdivision scheme defined in Section 2.3.2. In fact, for 
every function $\varphi \in C^{3}([-1,1])$, both differences $\varphi^{\prime}(-1)-2 \varphi^{\prime}(0)+\varphi^{\prime}(1)$ and $6\left(\frac{\varphi(1)-\varphi(-1)}{2}-\varphi^{\prime}(0)\right)$ are approximations of $\varphi^{(3)}(0)$.

The remaining coefficients in (11)

$$
\begin{aligned}
& \ell_{1}=C-B+\lambda \delta^{\prime}+\mu \delta, \quad r_{1}=C-B-\lambda \delta^{\prime}-\mu \delta, \\
& \ell_{2}=r_{2}=5 \delta / 2-\delta^{\prime} / 4, \\
& \ell_{3}=B-C / 2-2 \lambda \delta^{\prime}-2 \mu \delta, \quad r_{3}=B-C / 2+2 \lambda \delta^{\prime}+2 \mu \delta, \\
& \ell_{4}=r_{4}=\delta^{\prime} / 4-3 \delta / 2, \\
& C=p_{-1}-2 p_{0}+p_{1}, \quad B=\left(p_{1}^{\prime}-p_{-1}^{\prime}\right) / 4,
\end{aligned}
$$

are determined by solving two linear systems of equations derived from the remaining Hermite interpolating conditions $P_{H, \ell}(\alpha)=P_{H, r}(\alpha)=p_{\alpha}$ and $P_{H, \ell^{\prime}}(\alpha)=$ $P_{H, r}^{\prime}(\alpha)=p_{\alpha}^{\prime}$ for $\alpha \in\{-1,1\}$.

Remark 2 If $\pi \in \mathbb{P}_{2}$ and $p_{\alpha}=\pi(\alpha), p_{\alpha}^{\prime}=\pi^{\prime}(\alpha), \alpha \in\{-1,0,1\}$, then, by construction, the polynomial reproduction property of the Hermite interpolants is guaranteed and

$$
P_{H, \ell}=P_{H, r}=\pi .
$$

Moreover, for $\lambda=\mu=0$, Equation (13) holds for every $\pi \in \mathbb{P}_{5}$ interpolating the data at $\alpha \in\{-1,0,1\}$. Figure 2 (left), shows that for $(\lambda, \mu)=(0,0)$ the left and right interpolants coincide with the unique Hermite polynomial of degree 5 interpolating the data at $\alpha \in\{-1,0,1\}$. Therefore, if the data are sampled from a polynomial of degree 5 , both interpolants coincide with $\pi$. In other words, for $(\lambda, \mu)=(0,0)$, reproduction of degree 5 is guaranteed.

\subsubsection{Ternary Hermite subdivision scheme}

Similarly to Section 2.2 .2 , for $\boldsymbol{D}=\left(\begin{array}{cc}1 & 0 \\ 0 & 1 / 3\end{array}\right)$, to define the corresponding ternary Hermite subdivision scheme in (4), we choose the initial vector-valued sequence $\boldsymbol{f}_{0}=\left(\left(f_{0}(\alpha), f_{0}^{\prime}(\alpha)\right)^{T}, \alpha \in \mathbb{Z}\right)$ with

$$
f_{0}(\alpha)=p_{\alpha}, \quad f_{0}^{\prime}(\alpha)=p_{\alpha}^{\prime}, \quad \alpha \in\{-1,0,1\} \text { and } f_{0}(\alpha)=f_{0}^{\prime}(\alpha)=0, \quad \alpha \notin\{-1,0,1\},
$$
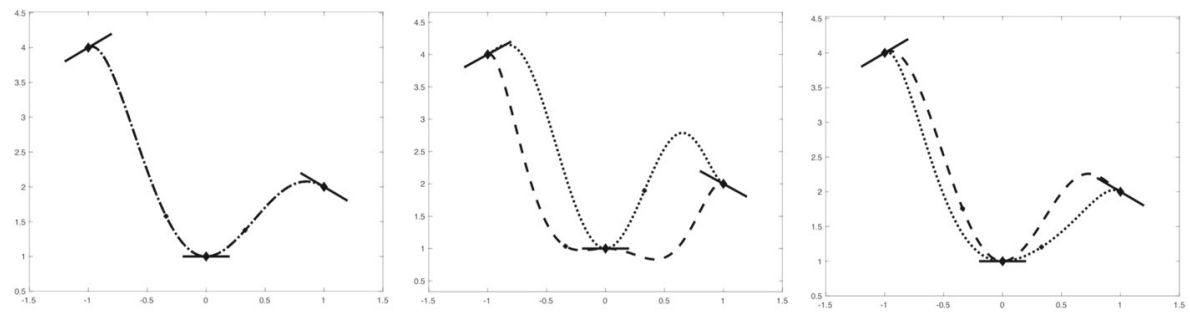

Fig. 2 Hermite interpolants $P_{H, \ell}$ (dashed) and $P_{H, r}$ (dotted) for $(\mu, \lambda)=(0,0),(2,6),(-6,-2)$, respectively, with corresponding evaluation points and derivatives 
and choose the sequence $\boldsymbol{f}_{1}=\left(\left(f_{1}(\alpha), f_{1}^{\prime}(\alpha)\right)^{T}, \alpha \in \mathbb{Z}\right)$ with

$$
\begin{array}{r}
f_{1}(-1)=P_{H, \ell}(-1 / 3), \quad f_{1}(0)=p_{0}, \quad f_{1}(1)=P_{H, r}(1 / 3), \\
f_{1}^{\prime}(-1)=P_{H, \ell}^{\prime}(-1 / 3), \quad f_{1}^{\prime}(0)=p_{0}^{\prime}, \quad f_{1}^{\prime}(1)=P_{H, r}^{\prime}(1 / 3) .
\end{array}
$$

Due to interpolation, we immediately deduce that

$$
\boldsymbol{A}(3 \alpha)=\delta_{0 \alpha} \boldsymbol{D}, \quad \alpha \in \mathbb{Z}
$$

To compute the other components of the mask $\boldsymbol{A}=(\boldsymbol{A}(\alpha), \alpha \in \mathbb{Z})$, we express the polynomials in (11) as

$$
P_{H, s}(t)=\sum_{\alpha \in\{-1,0,1\}} p_{\alpha} H_{\alpha, s, 0}(t)+\sum_{\alpha \in\{-1,0,1\}} p_{\alpha}^{\prime} H_{\alpha, s, 1}(t), \quad s \in\{\ell, r\},
$$

where the polynomials $H_{\alpha, s, k}$ for $\alpha \in\{-1,0,1\}$ and $k \in\{0,1\}$ are obtained from (11) imposing the cardinal Hermite interpolation conditions

$$
\begin{gathered}
H_{\alpha, s, 0}(\beta)=\delta_{\alpha \beta}, H_{\alpha, s, 0}^{\prime}(\beta)=0, \quad \beta \in\{-1,0,1\}, \quad s \in\{\ell, r\}, \\
H_{\alpha, s, 1}(\beta)=0, H_{\alpha, s, 1}^{\prime}(\beta)=\delta_{\alpha \beta}, \quad \beta \in\{-1,0,1\}, \quad s \in\{\ell, r\} .
\end{gathered}
$$

Then, for $s \in\{\ell, r\}$, the matrix form of the conditions on $\boldsymbol{f}_{1}$ at $\alpha_{\ell}=-1$ and $\alpha_{r}=1$ read as follows

$$
\begin{gathered}
\left(\begin{array}{l}
f_{1}\left(\alpha_{s}\right) \\
f_{1}^{\prime}\left(\alpha_{s}\right)
\end{array}\right)=\left(\begin{array}{ll}
H_{-1, s, 0}\left(\alpha_{s} / 3\right) & H_{-1, s, 1}\left(\alpha_{s} / 3\right) \\
H_{-1, s, 0}^{\prime}\left(\alpha_{s} / 3\right) & H_{-1, s, 1}^{\prime}\left(\alpha_{s} / 3\right)
\end{array}\right)\left(\begin{array}{l}
f_{0}(-1) \\
f_{0}^{\prime}(-1)
\end{array}\right) \\
+\left(\begin{array}{ll}
H_{0, s, 0}\left(\alpha_{s} / 3\right) & H_{0, s, 1}\left(\alpha_{s} / 3\right) \\
H_{0, s, 0}^{\prime}\left(\alpha_{s} / 3\right) & H_{0, s, 1}^{\prime}\left(\alpha_{s} / 3\right)
\end{array}\right)\left(\begin{array}{l}
f_{0}(0) \\
f_{0}^{\prime}(0)
\end{array}\right) \\
+\left(\begin{array}{ll}
H_{1, s, 0}\left(\alpha_{s} / 3\right) & H_{1, s, 1}\left(\alpha_{s} / 3\right) \\
H_{1, s, 0}^{\prime}\left(\alpha_{s} / 3\right) & H_{1, s, 1}^{\prime}\left(\alpha_{s} / 3\right)
\end{array}\right)\left(\begin{array}{l}
f_{0}(1) \\
f_{0}^{\prime}(1)
\end{array}\right) .
\end{gathered}
$$

The linear system corresponding to

$$
\boldsymbol{D} \boldsymbol{f}_{1}(\alpha)=\sum_{\beta \in(-1,0,1)} \boldsymbol{A}(\alpha-3 \beta) f_{0}(\beta), \quad \alpha \in\{-1,1\},
$$

for the chosen $f_{0}$ and $f_{1}$, uniquely identifies the remaining entries of the matrix mask $\boldsymbol{A}$ supported on $[-4,4]$ by taking into account that the grid spacing reduces 
at each subdivision step by $1 / 3$ (so that the factor $1 / 3$ appears when computing derivatives of (14))

$$
\begin{aligned}
& \boldsymbol{A}(-4)=\boldsymbol{D} \cdot \frac{1}{729}\left(\begin{array}{cc}
32 \mu+45 & 64 \lambda-12 \\
-144 \mu-162 & -288 \lambda+45
\end{array}\right), \quad \boldsymbol{A}(-3)=\left(\begin{array}{ll}
0 & 0 \\
0 & 0
\end{array}\right), \\
& \boldsymbol{A}(-2)=\boldsymbol{D} \cdot \frac{1}{729}\left(\begin{array}{cc}
-32 \mu+108 & -64 \lambda-24 \\
-144 \mu+702 & -288 \lambda-144
\end{array}\right) \text {, } \\
& \boldsymbol{A}(-1)=\boldsymbol{D} \cdot \frac{1}{729}\left(\begin{array}{c}
576-128 \lambda-64 \mu-192 \\
864576 \lambda+288 \mu+288
\end{array}\right), \\
& \boldsymbol{A}(0)=\boldsymbol{D}, \quad \boldsymbol{A}(1)=\boldsymbol{D} \cdot \frac{1}{729}\left(\begin{array}{cc}
576 & 128 \lambda+64 \mu+192 \\
-864 & 576 \lambda+288 \mu+288
\end{array}\right), \\
& \boldsymbol{A}(2)=\boldsymbol{D} \cdot \frac{1}{729}\left(\begin{array}{cc}
-32 \mu+108 & 64 \lambda+24 \\
144 \mu-702 & -288 \lambda-144
\end{array}\right), \\
& \boldsymbol{A}(3)=\left(\begin{array}{ll}
0 & 0 \\
0 & 0
\end{array}\right), \quad \boldsymbol{A}(4)=\boldsymbol{D} \cdot \frac{1}{729}\left(\begin{array}{cc}
32 \mu+45 & -64 \lambda+12 \\
144 \mu+162-288 \lambda+45
\end{array}\right) \text {. }
\end{aligned}
$$

Note that, by the support estimates in [8] which are also valid for Hermite schemes, the support of the basic limit function of the scheme defined in $(15)$ is $[-2,2]$.

\section{Regularity analysis of the Hermite family}

We recall that our motivation for the construction in Section 2 is a derivation of a class of ternary Hermite schemes in (15) of order $d=1$ which are at least $\mathcal{H C}^{2}$-smooth, for specific parameter pairs $(\mu, \lambda)$. To this purpose, we combine the joint spectral radius techniques in [4, 6, 7, 14] with the Taylor operator approach proposed in [27].

The main ingredients of our approach are listed below:

(i) The transition matrices derived from $\boldsymbol{A}$ in (15) and their polynomial reproduction properties, see Section 3.1.

(ii) The Taylor operator $S_{\boldsymbol{B}}$ associated with $S_{\boldsymbol{A}}$, the transition matrices derived from $\boldsymbol{B}$ and their polynomial reproduction properties, see Section 3.2.

(iii) The joint spectral radius analysis of $C^{2}$-regularity of $S_{A}$ and of $C^{0}$-regularity of $S_{\boldsymbol{B}}$, see Section 3.3.

(iv) Combination of $(i)-(i i i)$ for the $\mathcal{H C}^{2}$-regularity analysis of $H_{\mathbf{A}}$, see Section 3.4.

The regularity analysis in this section relies on joint spectral radius techniques, but does not provide any new contributions to the corresponding theory. Thus, we do not give an extended background on the joint spectral radius methods, but rather provide a detailed guide for the analysis steps in $(i)-(i v)$ and a summary of technicalities in the Appendix.

\subsection{Transition matrices from $A$}

The joint spectral radius approach connects the properties of subdivision operators with the properties of the corresponding transition matrices derived from subdivision masks [14]. The number of transition matrices is determined by the arity of the 
scheme. In the ternary case, we have three transition matrices derived from the matrix masks $\boldsymbol{A}$ in (15). They are defined by

$$
T_{\varepsilon}^{\boldsymbol{A}}=\left(\boldsymbol{A}^{T}(\varepsilon+3 \alpha-\beta)\right)_{\alpha, \beta \in \Omega^{A}} \quad \varepsilon \in\{0,1,2\},
$$

for an appropriate finite subset $\Omega^{A} \subset \mathbb{Z}$. Note that the choice of the transposed matrices in (16) is crucial for the existence of $\Omega^{A}$. The set $\Omega^{A}$ is computed using the Algorithm in [6, Lemma 3.8]. The Algorithm always terminates in finite number of steps and yields in this case $\Omega^{A}=\{-2,-1,0,1\}$ such that the finite sequences in $\ell\left(\Omega^{A}\right)$ are invariant under the associated transition operator (dual to $S_{\boldsymbol{A}}$ )

$$
\boldsymbol{g} \mapsto \sum_{\beta \in \mathbb{Z}} A^{T}(\varepsilon+3 \alpha-\beta) \boldsymbol{g}(\beta) .
$$

This invariance guarantees that all the properties of $S_{A}$ are encoded into the transition matrices in (16).

The transition matrices $T_{\varepsilon}^{A} \in \mathbb{R}^{8 \times 8}$ in (16) are $4 \times 4$ block matrices consisting of $2 \times 2$ matrix blocks $\boldsymbol{A}^{T}(\varepsilon+3 \alpha-\beta)$ read off the masks $\boldsymbol{A}$. By construction, the polynomial reproduction properties of $H_{A}$ summarized in Remark 2 guarantee that the polynomial sequences

$$
u_{0}=\left(\left(\begin{array}{l}
1 \\
0
\end{array}\right), \alpha \in \mathbb{Z}\right), u_{1}=\left(\left(\begin{array}{c}
\alpha \\
1
\end{array}\right), \alpha \in \mathbb{Z}\right) \text { and } u_{2}=\left(\left(\begin{array}{c}
\alpha^{2} \\
2 \alpha
\end{array}\right), \alpha \in \mathbb{Z}\right),
$$

are polynomial eigensequences of the subdivision operator $S_{A}$, i.e.

$$
S_{A} u_{m}=\left(\frac{1}{3}\right)^{m} u_{m}, \quad m=0,1,2 .
$$

The structure of $u_{0}$ indicates [13, Proposition 3.4] that the basic limit function $\Phi_{\boldsymbol{G}_{0}}$ of the scheme $S_{\boldsymbol{A}}$ has the form

$$
\Phi_{\boldsymbol{G}_{0}}=\lim _{n \rightarrow \infty} S_{A}^{n} \boldsymbol{G}_{0}=\left(\begin{array}{cc}
\phi_{1} & \phi_{2} \\
0 & 0
\end{array}\right), \quad \boldsymbol{G}_{0}=\delta I_{2},
$$

with $\phi_{1}$ and $\phi_{2}$ being the first components of the corresponding Hermite limits of $H_{\boldsymbol{A}}$ and $\delta$ is the Kronecker symbol centred at 0 . Therefore, due to Definition 2, one of the steps for showing that the Hermite scheme $H_{A}$ is $\mathcal{H C}^{2}$-smooth is to show that $\phi_{1}, \phi_{2}$ belong to $C^{2}(\mathbb{R})$.

By [1, Theorem 3.1], the eigenproperties of $S_{A}$ in (17) imply that the subspaces of $\mathbb{R}^{8}$ spanned by the following three column vectors, obtained by restricting $u_{0}, u_{1}$ and $u_{2}$, respectively, to $\Omega^{A}$,

$$
\left(\begin{array}{l}
1 \\
0 \\
1 \\
0 \\
1 \\
0 \\
1 \\
0
\end{array}\right), \quad\left(\begin{array}{r}
-2 \\
1 \\
-1 \\
1 \\
0 \\
1 \\
1 \\
1
\end{array}\right) \text { and } \quad\left(\begin{array}{r}
4 \\
-4 \\
1 \\
-2 \\
0 \\
0 \\
1 \\
2
\end{array}\right)
$$


are right-invariant under all $T_{\varepsilon}^{\boldsymbol{A}}, \varepsilon \in\{0,1,2\}$, and the difference subspaces $V_{0}^{\boldsymbol{A}}, V_{1}^{\boldsymbol{A}}$ and $V_{2}^{A}$

$$
V_{0}^{A}=\left(\begin{array}{rrrrrrr}
1 & 0 & 0 & 0 & 0 & 0 & 0 \\
0 & 0 & 0 & 1 & 0 & 0 & 0 \\
-1 & 1 & 0 & 0 & 0 & 0 & 0 \\
0 & 0 & 0 & 0 & 1 & 0 & 0 \\
0 & -1 & 1 & 0 & 0 & 0 & 0 \\
0 & 0 & 0 & 0 & 0 & 1 & 0 \\
0 & 0 & -1 & 0 & 0 & 0 & 0 \\
0 & 0 & 0 & 0 & 0 & 0 & 1
\end{array}\right), \quad V_{1}^{A}=\left(\begin{array}{rrrrrr}
1 & 0 & 0 & 0 & 0 & 1 \\
0 & 0 & 1 & 0 & 0 & -1 \\
-2 & 1 & 0 & 0 & 0 & -1 \\
0 & 0 & -1 & 1 & 0 & 0 \\
1 & -2 & 0 & 0 & 0 & 0 \\
0 & 0 & 0 & -1 & 1 & 0 \\
0 & 1 & 0 & 0 & 0 & 0 \\
0 & 0 & 0 & 0 & -1 & 0
\end{array}\right),
$$

and

$$
V_{2}^{A}=\left(\begin{array}{rrrrr}
1 & 0 & 0 & -2 & 0 \\
0 & 1 & 0 & 1 & -1 \\
-3 & 0 & 0 & 2 & -2 \\
0 & -2 & 1 & 1 & 3 \\
3 & 0 & 0 & 0 & 2 \\
0 & 1 & -2 & 0 & 0 \\
-1 & 0 & 0 & 0 & 0 \\
0 & 0 & 1 & 0 & 0
\end{array}\right)
$$

satisfying their defining property

$$
\sum_{\alpha \in \mathbb{Z}} v(\alpha) u_{j}(-\alpha)=0, \quad v \in V_{k}^{A}, \quad 0 \leq j \leq k, \quad k \in\{0,1,2\},
$$

are left-invariant under $T_{\varepsilon}^{\boldsymbol{A}}, \varepsilon \in\{0,1,2\}$. Since we are interested in the $C^{2}$ regularity of $S_{A}$, in Proposition 1 below, we restrict the transition matrices $T_{\varepsilon}^{A}, \varepsilon \in\{0,1,2\}$ to $V_{2}^{A}$. This restriction is done using the standard linear algebra techniques and mimics taking the second-order differences of the limit functions $\phi_{1}$ and $\phi_{2}$. For the interested reader, we give the restricted matrices $\left\{\left.T_{\varepsilon}^{A}\right|_{V_{2}^{A}}: \varepsilon \in\{0,1,2\}\right\}$ in (26) in the Appendix.

\subsection{Taylor subdivision operator $S_{B}$ and transition matrices from $B$}

In this section, we construct the Taylor operator $S_{\boldsymbol{B}}$ for $H_{\boldsymbol{A}}$ and derive the corresponding transition matrices from the mask $\boldsymbol{B}$. The Taylor operator was proposed in [27] and more recently generalized in [29] and [32]. Note that, even if the results in [27] are only given for the binary schemes, there is no substantial difference in case of ternary schemes. The Taylor operator relates the symbols of $\boldsymbol{A}$ and $\boldsymbol{B}$ by the following identity

$$
\left(\begin{array}{rr}
z-1 & -1 \\
0 & 1
\end{array}\right) \boldsymbol{A}^{*}(z)=\frac{1}{3} \boldsymbol{B}^{*}(z)\left(\begin{array}{rr}
z^{3}-1 & -1 \\
0 & 1
\end{array}\right), \quad z \in \mathbb{C} \backslash(0),
$$

where $\boldsymbol{A}^{*}(z)$ is the symbol associated with the subdivision operator $S_{\boldsymbol{A}}$ and $\boldsymbol{B}^{*}(z)$ the symbol associated with the Taylor operator $S_{\boldsymbol{B}}$. In our case, the computation of 
the symbol $\boldsymbol{B}^{*}(z)$ yields the mask $\boldsymbol{B}$ given in (28) in the Appendix. From the mask $\boldsymbol{B}$, similarly to Section 3.1, we define the transition matrices

$$
T_{\varepsilon}^{\boldsymbol{B}}=\left(\boldsymbol{B}^{T}(\varepsilon+3 \alpha-\beta)\right)_{\alpha, \beta \in \Omega^{\boldsymbol{B}}} \quad \varepsilon \in\{0,1,2\} \quad \Omega^{\boldsymbol{B}}=\{-3,-2,-1,0,1\} .
$$

These transition matrices $T_{\varepsilon}^{\boldsymbol{B}} \in \mathbb{R}^{10 \times 10}$ are $5 \times 5$ block matrices consisting of $2 \times 2$ matrix blocks $\boldsymbol{B}^{T}(\varepsilon+3 \alpha-\beta)$ defined by the mask $\boldsymbol{B}$ in (28).

The polynomial reproduction property of $H_{\boldsymbol{A}}$ in Remark 2 guarantees that the polynomial sequence

$$
u_{0}=\left(\left(\begin{array}{r}
-2 \\
1
\end{array}\right), \alpha \in \mathbb{Z}\right),
$$

is a polynomial eigensequence of the subdivision operator $S_{\boldsymbol{B}}$, i.e. $S_{\boldsymbol{B}} u_{0}=u_{0}$. The size and the structure of the common difference subspace $V_{0}^{\boldsymbol{B}}$ of the matrices in (18) orthogonal to the constant sequence $u_{0}$ restricted to $\Omega^{\boldsymbol{B}}$ are identified by the column vectors

$$
V_{0}^{\boldsymbol{B}}=\left(\begin{array}{rrrrrrr}
1 & 0 & 0 & 0 & 0 & 0 & 0 \\
0 & 1 & 0 & 0 & 0 & 0 & 0 \\
-2 & 0 & 1 & 0 & 0 & 0 & 0 \\
0 & -2 & 0 & 1 & 0 & -1 & 1 \\
1 & 0 & -2 & 0 & 0 & -1 & 3 \\
0 & 1 & 0 & -2 & 1 & 0 & 3 \\
0 & 0 & 1 & 0 & 0 & 1 & -1 \\
0 & 0 & 0 & 1 & -2 & 1 & 0 \\
0 & 0 & 0 & 0 & 0 & 0 & 0 \\
0 & 0 & 0 & 0 & 1 & 0 & 0
\end{array}\right) .
$$

$V_{0}^{\boldsymbol{B}}$ is left-invariant under all transition matrices $T_{\varepsilon}^{\boldsymbol{B}}, \varepsilon \in\{0,1,2\}$. Since we are interested in the $C^{0}$-regularity of $S_{\boldsymbol{B}}$, in Proposition 2 below, we analyze the joint spectral properties of the matrix set $\left\{\left.T_{\varepsilon}^{\boldsymbol{B}}\right|_{V_{0}^{B}}: \varepsilon \in\{0,1,2\}\right\}$. This matrix set is given in (27) in the Appendix.

\subsection{Joint spectral radius analysis of $S_{A}$ and $S_{B}$}

The joint spectral radius approach for regularity analysis of scalar binary refinable functions was introduced in [14] and has been generalized to various different scenarios since then. This approach is based on the concept of the joint spectral radius

$$
\rho(\mathcal{T})=\lim _{n \rightarrow \infty} \max _{T_{j_{k}} \in \mathcal{T}}\left\|\prod_{k=1}^{n} T_{j_{k}}\right\|^{1 / n}
$$

introduced for finite matrix sets $\mathcal{T}=\left\{T_{j} \in \mathbb{R}^{n \times n}: j=1, \ldots, J\right\}, J \in \mathbb{N}$, in [33].

The limit in (20) exists and is independent of the matrix norm. It is well known that the joint spectral radius can be used to measure the joint contractivity of the matrices in $\mathcal{T}$, see e.g. (22) in the proof of Proposition 1.

The recent advances in numerical linear algebra allow for exact computations of the joint spectral radius of finite sets of transition matrices restricted to common difference subspaces [19, 20, 31]. Thus, providing vigorous mathematical proofs for 
convergence and regularity of subdivision schemes. Moreover, we are able to treat the parameter dependency of the transition matrices by the techniques presented in [4, Theorem 3.2, Remark 3.5 (iii)].

\subsubsection{Parameter domains for $C^{2}$-regularity of $S_{A}$ and for $C^{0}$-regularity of $S_{B}$}

In this subsection, in Proposition 1, we prove that $S_{A}$ is $C^{2}$-smooth for $(\mu, \lambda) \in K_{2}$ and, in Proposition 2, that $S_{\boldsymbol{B}}$ in (28) is convergent for $(\mu, \lambda) \in K_{0}$.

The polygonal set $K_{2} \subset \mathbb{R}^{2}$ on Fig. 3 is computed using the algorithms in [19, 20, $31]$ and is the closed convex hull

$$
K_{2}=\operatorname{co}\left\{\left(\mu_{2, m}, \lambda_{2, m}\right) \in \mathbb{R}^{2}: m=1, \ldots, 6\right\}
$$

of the points

$$
\begin{aligned}
& \left(\mu_{2,1}, \lambda_{2,1}\right)=(-271 / 100, \quad 519 / 971), \quad\left(\mu_{2,2}, \lambda_{2,2}\right)=(-159 / 73, \quad 78 / 175), \\
& \left(\mu_{2,3}, \lambda_{2,3}\right)=(-2417 / 2062, \quad 509 / 2217), \quad\left(\mu_{2,4}, \lambda_{2,4}\right)=(-185 / 91, \quad 92 / 293), \\
& \left(\mu_{2,5}, \lambda_{2,5}\right)=(-271 / 100, \quad 311 / 661), \quad\left(\mu_{2,6}, \lambda_{2,6}\right)=(-277 / 100, \quad 113 / 222) \text {. }
\end{aligned}
$$

Proposition 1 The scheme $S_{\boldsymbol{A}}$ in (15) is $\mathcal{C}^{2}$-smooth for $(\mu, \lambda) \in K_{2}$.

Proof To stress the parameter dependence, we define

$$
\left.T_{\varepsilon, \mu, \lambda}^{\boldsymbol{A}}\right|_{V_{2}^{A}}=\left.T_{\varepsilon}^{\boldsymbol{A}}\right|_{V_{2}^{A}} \quad \text { for } \quad \varepsilon \in\{0,1,2\} .
$$

Define $U=\operatorname{span}\left\{u_{1}, u_{2}, u_{3}\right\}$ with $u_{j}, j=1,2,3$, in (17) and note that the columns of $V_{2}^{A}$ in Section 3.1 can be identified with sequences in $\ell^{2 \times 1}\left(\Omega^{A}\right)$ for $\Omega^{A}=\{-2,-1,0,1\}$. Then, straightforward computations show that

$$
\sum_{\alpha \in \mathbb{Z}} v(\alpha) u(-\alpha)=0, \quad v \in V_{2}^{A}, \quad u \in U
$$

This orthogonality property of the second differences in $V_{2}^{A}$ states that the restricted matrices in

$$
\mathcal{T}^{\boldsymbol{A}}=\left\{\left.T_{\varepsilon, \mu, \lambda}^{\boldsymbol{A}}\right|_{V_{2}^{\boldsymbol{A}}}: \varepsilon \in\{0,1,2\},(\mu, \lambda) \in K_{2}\right\},
$$

indeed contain all the information about the $\mathcal{C}^{2}$-smoothness of $S_{\boldsymbol{A}}$ for $(\mu, \lambda) \in$ $K_{2}$. Therefore, due to the minimality of $\Omega^{A}$ and by [25, Theorem 4.1] which characterizes the smoothness of $S_{\boldsymbol{A}}$ in terms of $\rho\left(\mathcal{T}^{\boldsymbol{A}}\right)$, it suffices to show that

$$
\rho\left(\mathcal{T}^{\boldsymbol{A}}\right)<3^{-2} \text {. }
$$

However, it is an impossible task to check this joint contractivity of the matrices in $\mathcal{T}^{A}$ for all pairs $(\mu, \lambda) \in K_{2}$. Instead, we use the results in [4, Theorem 3.2, Remark 3.5 (iii)] which state that the contractivity $\rho\left(\mathcal{T}^{\boldsymbol{A}}\right)<3^{-2}$ for all parameter pairs inside of any convex domain is implied by the joint contractivity of all restricted transition matrices defined by the corners of this domain. This joint contractivity unfortunately is not achieved for the corners of $K_{2}$ and, therefore, we partition $K_{2}$ into appropriate 
convex subdomains. We decided to use the well-known Delauney triangulation $\Delta$ of $K_{2}$

$$
\Delta=\left\{\Delta_{j}=\operatorname{co}\left\{k_{j}^{1}, k_{j}^{2}, k_{j}^{3}\right\}: k_{j}^{m} \in K_{2}, m=1,2,3, j=1, \ldots, J\right\}, \quad J \in \mathbb{N},
$$

with vertices $k_{j}^{m} \in K_{2}$. Next, for each triangular subdomain $\Delta_{j} \in \Delta$, we define the following set of nine (for all three corners of $\Delta_{j}$ ) transition matrices

$$
\mathcal{T}_{j}^{\boldsymbol{A}}=\left\{\left.T_{\varepsilon, \mu, \lambda}^{\boldsymbol{A}}\right|_{V_{2}^{\boldsymbol{A}}}: \varepsilon \in\{0,1,2\},(\mu, \lambda)=k_{j}^{m}, m=1,2,3\right\} .
$$

As mentioned above by [4, Theorem 3.2, Remark 3.5 (iii)], if

$$
\rho\left(\mathcal{T}_{j}^{\boldsymbol{A}}\right)<3^{-2}
$$

then, for each parameter pair $(\mu, \lambda) \in \Delta_{j}$,

$$
\rho\left(\mathcal{T}_{\Delta_{j}}^{\boldsymbol{A}}\right)<3^{-2} \text { with } \mathcal{T}_{\Delta_{j}}=\left\{\left.T_{\varepsilon, \mu, \lambda}^{\boldsymbol{A}}\right|_{V_{2}^{A}}: \varepsilon \in\{0,1,2\}\right\} .
$$

To prove (22) for $j=1, \ldots, J$, we use the results in $[19,20]$ as well as the modified Gripenberg and modified invariant polytope algorithms in [31]. For each triangle $\Delta_{j}$ in the triangulation $\Delta$ of $K_{2}$, or for its dyadically refined version, the algorithms terminate successfully determining the spectrum maximizing matrix product from $\mathcal{T}_{j}^{A}$. This proves (22) for all $j=1, \ldots, J$ and, thus, the claim follows.

Remark 3 Note that the use of the triangulation $\Delta$ of $K_{2}$ in the proof of Proposition 1 is unavoidable. In fact, if we compute the joint spectral radius of a matrix set defined by the vertices of the whole domain $K_{2}$ the sufficient conditions in [4, Theorem 3.2, Remark 3.5 (iii)] are not satisfied.

The regularity analysis of $S_{\boldsymbol{A}}$ is not sufficient to conclude $\mathcal{H C}^{2}$-regularity of $\mathcal{H}_{\boldsymbol{A}}$. Therefore, we proceed with the analysis of the Taylor operator $S_{\boldsymbol{B}}$ in (28) for parameter values $(\mu, \lambda) \in K_{0}$.

The set $K_{0} \subset \mathbb{R}^{2}$ on Fig. 3 is the closed convex hull

$$
K_{0}=\operatorname{co}\left\{\left(\mu_{0, m}, \lambda_{0, m}\right) \in \mathbb{R}^{2}: m=1, \ldots, 7\right\}
$$

of the points

$$
\begin{array}{rrrr}
\left(\mu_{0,1}, \lambda_{0,1}\right)=\left(\begin{array}{lr}
-513 / 50, & 231 / 50
\end{array}\right), & \left(\mu_{0,2}, \lambda_{0,2}\right)=\left(\begin{array}{ll}
357 / 125, & 216 / 125
\end{array}\right), \\
\left(\mu_{0,3}, \lambda_{0,3}\right)=\left(\begin{array}{lr}
230 / 50, & 310 / 250
\end{array}\right), & \left(\mu_{0,4}, \lambda_{0,4}\right)=\left(\begin{array}{ll}
520 / 129, & -748 / 217
\end{array}\right), \\
\left(\mu_{0,5}, \lambda_{0,5}\right)=\left(\begin{array}{lrl}
-230 / 50, & -301 / 200
\end{array}\right), & \left(\mu_{0,6}, \lambda_{0,6}\right)=\left(\begin{array}{ll}
-950 / 80, & 403 / 100
\end{array}\right), \\
\left(\mu_{0,7}, \lambda_{0,7}\right)=\left(\begin{array}{lll}
-627 / 53, & 211 / 50
\end{array}\right) &
\end{array}
$$

Proposition 2 The scheme $S_{\boldsymbol{B}}$ in (28) is convergent for $(\mu, \lambda) \in K_{0}$.

Proof The proof mimics the proof of Proposition 1 by replacing the transition matrices of $\boldsymbol{A}$ by the one in (27). 


\subsubsection{Parameter domains for $\mathcal{C}^{0}$ and $\mathcal{C}^{1}$-regularity of $S_{A}$}

For completeness, we also describe the parameter domains $K_{0}$ and $K_{1}$ for $\mathcal{C}^{0}$ and $\mathcal{C}^{1}$ convergence of $S_{A}$, respectively. They are depicted in Fig. 3. The domain $K_{0}$ is the one in (23) while the domain $K_{1}$ is obtained using the argument similar to the one in Proposition 1. More in details, the domain $K_{1}$ is a closed convex hull

$$
K_{1}=\operatorname{co}\left\{\left(\mu_{1, m}, \lambda_{1, m}\right) \in \mathbb{R}^{2}: m=1, \ldots, 7\right\}
$$

of the points

$$
\begin{aligned}
& \left(\mu_{1,1}, \lambda_{1,1}\right)=(-51 / 10, \quad 31 / 22), \quad\left(\mu_{1,2}, \lambda_{1,2}\right)=(-159 / 73, \quad 9 / 7), \\
& \left(\mu_{1,3}, \lambda_{1,3}\right)=(4 / 5, \quad 3 / 8), \quad\left(\mu_{1,4}, \lambda_{1,4}\right)=(13 / 14, \quad 6 / 25), \\
& \left(\mu_{1,5}, \lambda_{1,5}\right)=(-13 / 8, \quad-7 / 20), \quad\left(\mu_{1,6}, \lambda_{1,6}\right)=(-19 / 8, \quad-2 / 13) \text {, } \\
& \left(\mu_{1,7}, \lambda_{1,7}\right)=(-59 / 11, \quad 9 / 7) \text {. }
\end{aligned}
$$

\subsection{Parameter domain for $\mathcal{H C}^{2}$-regularity analysis of $H_{A}$}

The main result of Section 3, Theorem 1, shows that the Hermite scheme $H_{\boldsymbol{A}}$ in (15) is $\mathcal{H C}^{2}$-smooth for $(\mu, \lambda) \in K_{2}$. The proof of Theorem 1 is based on Proposition 1 and Proposition 2 in the previous subsection. Indeed, combining them we are finally ready to prove the main result of this paper.

Theorem 1 The scheme $H_{A}$ in (15) is $\mathcal{H C}^{2}$-smooth for $(\mu, \lambda) \in K_{2}$.

Proof Since, by Proposition $2, H_{A}$ is convergent its vector-valued limit functions $\boldsymbol{\Phi}: \mathbb{R} \rightarrow \mathbb{R}^{2}$, has structure $\boldsymbol{\Phi}=\left(\begin{array}{c}\phi \\ \phi^{\prime}\end{array}\right)$ with $\phi^{\prime} \in C^{0}$. On the other hand, since, by

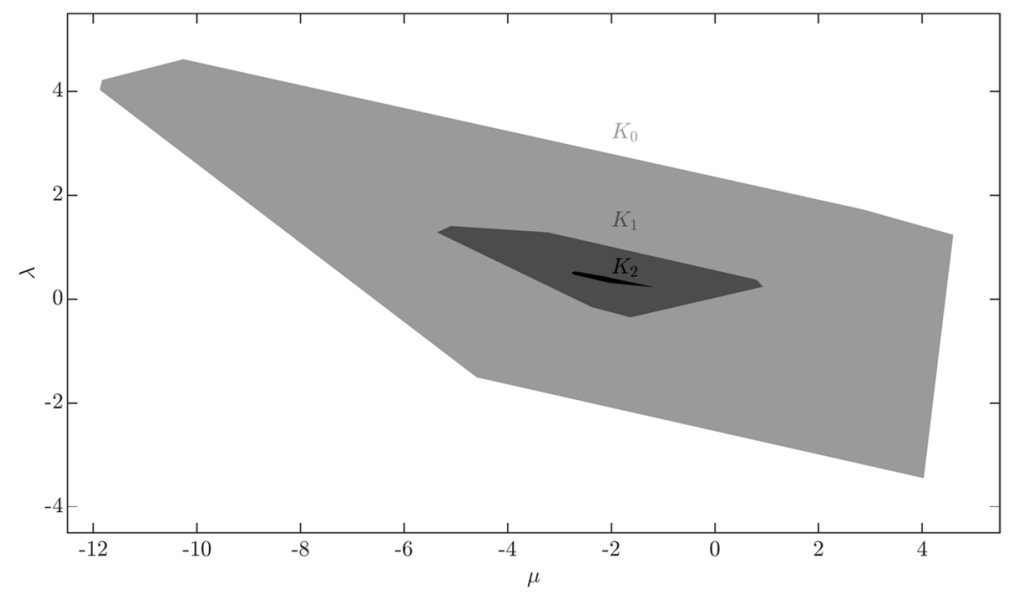

Fig. 3 Parameter domains $K_{2} \subset K_{1} \subset K_{0}$ 
Proposition $1, S_{A}$ is $C^{2}$-convergent it follows that $\phi \in C^{2}$. Hence $\phi^{\prime} \in C^{1}$, which implies the $\mathcal{H C}^{2}$-convergence of $H_{\boldsymbol{A}}$.

\section{Complete Taylor operator approach for the extended scheme}

Usually the visual quality of subdivision limits is determined by both the contractivity of the corresponding difference schemes and by the Hölder exponent of the corresponding limit. In this section, we search for the parameter pair $\left(\mu^{*}, \lambda^{*}\right) \in K_{2}$ such that both of these properties are optimal in the following sense. We construct the difference scheme $S_{\tilde{\boldsymbol{B}}_{+}}$of the extended Hermite scheme $H_{\boldsymbol{A}_{+}}$in (24) and find $\left(\mu^{*}, \lambda^{*}\right) \in K_{2}$ such that the difference scheme is contractive $\left\|S_{\tilde{\boldsymbol{B}}_{+}}^{R}\right\|_{\infty}<1$ with the smallest possible $R$. At the same time $\left(\mu^{*}, \lambda^{*}\right) \in K_{2}$ should define a Hermite scheme $H_{A}$ such that its limits possess a high Hölder exponent. We call such a parameter pair $\left(\mu^{*}, \lambda^{*}\right) \in K_{2}$ optimal and claim that the corresponding Hermite scheme $H_{A}$ yields visually smoother curves in fewer iterations, see Fig. 4.

To determine the optimal parameters $\left(\mu^{*}, \lambda^{*}\right) \in K_{2}$, we derive the complete Taylor factorization [27] of the extended Hermite scheme $H_{A+}$ in (24) of order 2 and study its contractivity for different parameter values $(\mu, \lambda) \in K_{2}$.
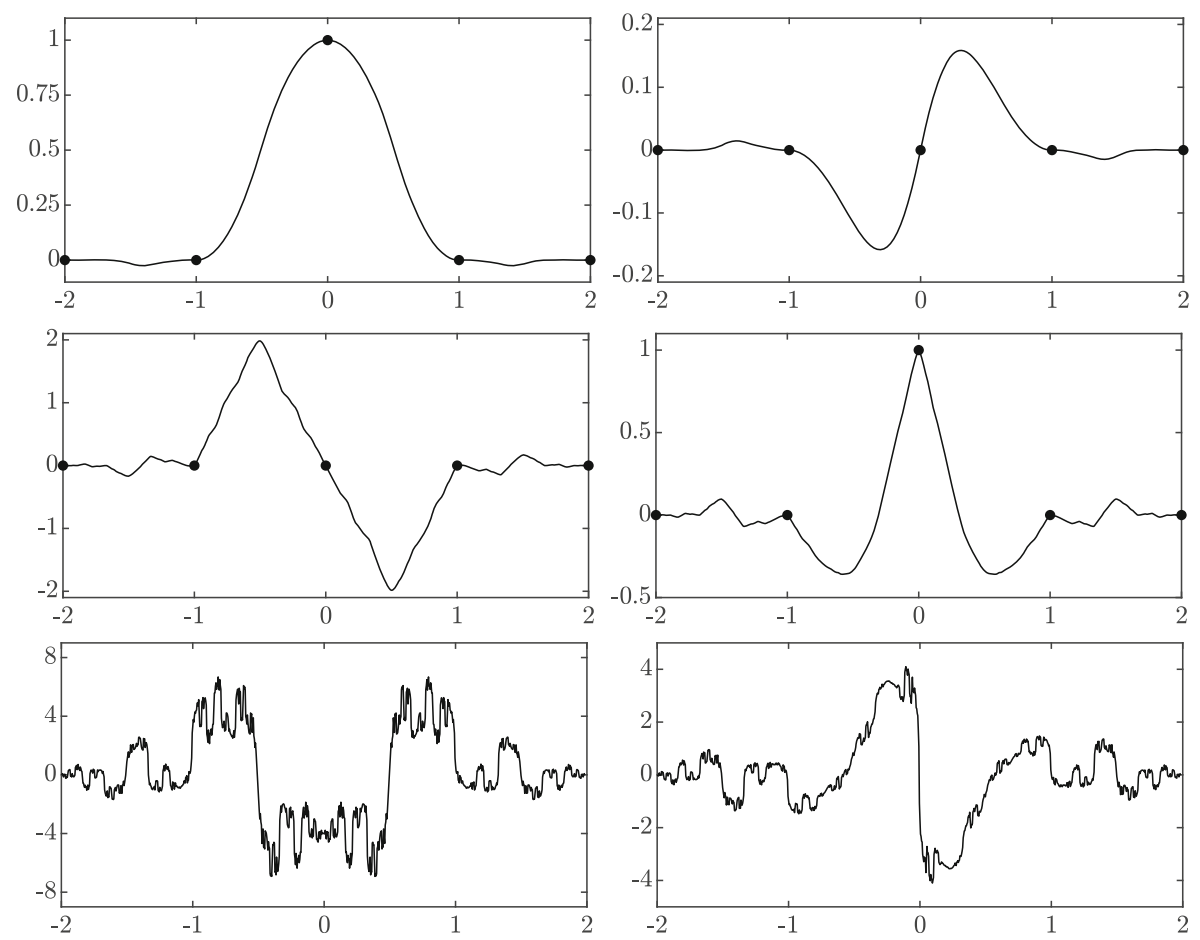

Fig. 4 Basic limit functions $\phi_{1}$ and $\phi_{2}$, and their first two derivatives, corresponding to the parameters

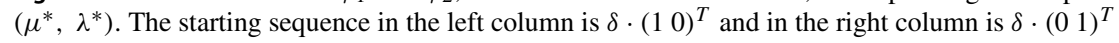


To define the scheme $H_{A+}$ we use the results from [27] and [28] and start by completing the sequences $f_{n} \in \ell^{2}(\mathbb{Z})$ generated by $H_{A}$ by one additional component determined from the following approximations of the second derivative. In particular, for $\varphi \in C^{3}(\mathbb{R})$ and for small $h \in \mathbb{R}$, we use the following approximations

$$
\begin{aligned}
\varphi^{\prime \prime}(x) & \approx \frac{1}{2 h}\left(\varphi^{\prime}(x+h)-\varphi^{\prime}(x-h)\right), \\
\varphi^{\prime \prime}(x+h / 3) & \approx \frac{1}{6 h}\left(5 \varphi^{\prime \prime}(x+h)-4 \varphi^{\prime \prime}(x)-\varphi^{\prime \prime}(x-h)\right), \quad x \in \mathbb{R}, \\
\varphi^{\prime \prime}(x-h / 3) & \approx \frac{1}{6 h}\left(-5 \varphi^{\prime}(x-h)+4 \varphi^{\prime}(x)+\varphi^{\prime}(x+h)\right) .
\end{aligned}
$$

Note that for an arbitrary initial sequence $\left(f_{0}(\alpha)=\left(f_{0}(\alpha) f_{0}^{\prime}(\alpha)\right)^{T}, \alpha \in \mathbb{Z}\right) \in$ $\ell^{2}(\mathbb{Z})$, at the $n$th step of the subdivision recursion, the above approximations suggest the construction of a new sequence in $\ell^{3}(\mathbb{Z})$ with additional component $f_{n+1}^{\prime \prime}(\alpha)$ for $\alpha \in \mathbb{Z}$ defined by

$$
\begin{aligned}
3^{-2(n+1)} f_{n+1}^{\prime \prime}(3 \alpha) & =\frac{3^{-2}}{2}\left(3^{-n} f_{n}^{\prime}(\alpha+1)-3^{-n} f_{n}^{\prime}(\alpha-1)\right), \\
3^{-2(n+1)} f_{n+1}^{\prime \prime}(3 \alpha+1) & =\frac{3^{-3}}{2}\left(5 \cdot 3^{-n} f_{n}^{\prime}(\alpha+1)-4 \cdot 3^{-n} f_{n}^{\prime}(\alpha)-3^{-n} f_{n}^{\prime}(\alpha-1)\right), \\
3^{-2(n+1)} f_{n+1}^{\prime \prime}(3 \alpha-1) & =\frac{3^{-3}}{2}\left(-5 \cdot 3^{-n} f_{n}^{\prime}(\alpha-1)+4 \cdot 3^{-n} f_{n}^{\prime}(\alpha)+3^{-n} f_{n}^{\prime}(\alpha+1)\right) .
\end{aligned}
$$

These identities define additional subdivision rules for the scheme of type (4) with the corresponding subdivision mask $\boldsymbol{A}_{+} \in \ell^{3 \times 3}(\mathbb{Z})$ given by its symbol

$$
\boldsymbol{A}_{+}^{*}(z)=\sum_{\alpha \in \mathbb{Z}} \boldsymbol{A}_{+}(\alpha) z^{\alpha}=\left(\begin{array}{ccc}
a_{11}(z) & a_{12}(z) & 0 \\
a_{21}(z) & a_{22}(z) & 0 \\
0 & q_{2}(z) & 0
\end{array}\right), \quad z \in \mathbb{C} \backslash(0),
$$

with

$$
\left(\begin{array}{ll}
a_{11}(z) & a_{12}(z) \\
a_{21}(z) & a_{22}(z)
\end{array}\right)=\sum_{\alpha \in \mathbb{Z}} \boldsymbol{A}(\alpha) z^{\alpha}
$$

defined by using (15) and with

$$
q_{2}(z)=-\frac{z^{-4}-z^{4}+3\left(z^{-3}-z^{3}\right)+5\left(z^{-2}-z^{2}\right)+4\left(z^{-1}-z\right)}{54}, \quad z \in \mathbb{C} \backslash(0) .
$$

The complete Taylor factorization of $\boldsymbol{A}_{+}^{*}(z)$ defines the operator $S_{\tilde{\boldsymbol{B}}_{+}}: \ell^{3}(\mathbb{Z}) \rightarrow$ $\ell^{3}(\mathbb{Z})$ whose symbol $\tilde{\boldsymbol{B}}_{+}^{*}(z)$ (given in Appendix) satisfies

$$
\left(\begin{array}{rrr}
z^{-1}-1 & -1 & -1 / 2 \\
0 & z^{-1}-1 & -1 \\
0 & 0 & z^{-1}-1
\end{array}\right) \boldsymbol{A}_{+}^{*}(z)=\frac{1}{9} \tilde{\boldsymbol{B}}_{+}^{*}(z)\left(\begin{array}{rrr}
z^{-3}-1 & -1 & -1 / 2 \\
0 & z^{-3}-1 & -1 \\
0 & 0 z^{-3}-1
\end{array}\right)
$$


By [27], the scheme $H_{\boldsymbol{A}}$ is $\mathcal{H C}^{2}$-smooth, if the stationary vector subdivision scheme $S_{\tilde{\mathcal{B}}_{+}}$is contractive, i.e there exists $R \in \mathbb{N}$ such that $\left\|S_{\tilde{\boldsymbol{B}}_{+}}^{R}\right\|_{\infty}<1$. By [16], the latter is equivalent to show that

$$
\max \left\{\left\|\sum_{\beta \in \mathbb{Z}}\left|\tilde{\boldsymbol{B}}_{+}^{[R]}(\varepsilon-3 \beta)\right|\right\|_{\infty}, \quad \varepsilon \in\{0,1,2\}\right\}<1,
$$

with the $R$-iterated mask $\widetilde{\boldsymbol{B}}_{+}^{[R]}$ computed by the iteration

$$
\tilde{\boldsymbol{B}}_{+}^{[1]}=\tilde{\boldsymbol{B}}_{+}, \quad \tilde{\boldsymbol{B}}_{+}^{[r]}=\sum_{\beta \in \mathbb{Z}} \tilde{\boldsymbol{B}}_{+}(\cdot-3 \beta) \tilde{\boldsymbol{B}}_{+}^{[r-1]}(\beta), \quad r=1, \ldots, R
$$

Matlab computations for

$$
\begin{aligned}
\mu=-1.9066, & \lambda=0.33394, \\
\mu^{*}=-1.8630, & \lambda^{*}=0.32874,
\end{aligned}
$$

yield the results presented in the following table where $R$ is the smallest integer such that $\left\|S_{S_{\tilde{\boldsymbol{B}}_{+}}^{R}}^{R}\right\|_{\infty}<1$; The real value $\alpha_{\phi} \geq-\log _{3}(\rho(\mathcal{T}))$ is the Hölder exponent of $\phi$, the first component of the vector limit function, while s.m.p. is the spectrum maximizing product $\prod$ of length $n$ that attains the joint spectral radius, i.e. the joint spectral radius $\rho(\mathcal{T})=\rho\left(\prod\right)^{1 / n}$.

\begin{tabular}{lllll}
\hline & $R$ & $\left\|S_{\tilde{\boldsymbol{B}}}^{R}\right\|_{\infty}$ & $\alpha_{\phi}$ & s.m.p. length \\
\hline$(\mu, \lambda)$ & 10 & 0.68288 & $2.40722 \ldots$ & 19 \\
$\left(\mu^{*}, \lambda^{*}\right)$ & 9 & 0.72294 & $2.40286 \ldots$ & 18 \\
\hline
\end{tabular}

\section{Conclusion}

The geometric interpretation of the ternary interpolatory scalar three-point subdivision scheme by Hassan and Dodgson allows us to derive a family of ternary interpolatory Hermite subdivision scheme with high smoothness and short support. The construction is based on the local use of two interpolating Hermite polynomials and their evaluation at the points of a ternary grid. The analysis of this new family of Hermite scheme, depending on two parameters, is conducted by combining Taylor operator and joint spectral radius techniques. Since the latter was not yet used for regularity analysis of Hermite schemes, we provide a detailed description of the necessary steps for its use. We are able to identify a parameter region for $\mathcal{H C}^{2}$-regularity, higher than usually expected for Hermite schemes of order 1. 
The proposed family of ternary Hermite schemes shows the hidden relation between the arity of the scheme and its smoothness. However, at the moment, we do not have a complete picture of this connection. The theory linking arity, order and smoothness of a Hermite scheme is a subject for further investigation.

\section{Appendix}

For the interested reader, we provide the details of our MATLAB routines via MATLAB exchange, see t-toolboxes [30]. For completeness, we provide here the transition matrices $\left\{\left.T_{\varepsilon}^{\boldsymbol{A}}\right|_{V_{2}^{\boldsymbol{A}}}: \varepsilon \in\{0,1,2\}\right\}$

$$
\begin{aligned}
& \frac{1}{729}\left(\begin{array}{ccccc}
64 \mu+243 & 96 \mu+108 & 180-96 \mu & -112 \mu-144 & -80 \mu-72 \\
64 \lambda-128 \mu-480 & -96 \lambda-192 \mu-57 & 192 \mu-360 & 224 \mu-224 \lambda+462 & 96 \lambda+160 \mu-6 \\
128 \lambda-60 & 192 \lambda-30 & -192 \lambda-33 & 39-224 \lambda & 21-160 \lambda \\
16 \mu+99 & 72 \mu+81 & 90-48 \mu & 0 & -64 \mu-63 \\
-112 \mu-369 & 9-120 \mu & 144 \mu-270 & 224 \mu+423 & 96 \mu-54
\end{array}\right), \\
& \frac{1}{729}\left(\begin{array}{ccccc}
-96 \mu-135 & -48 \mu-54 & 96 \mu+108 & 0 & -64 \mu-90 \\
192 \mu-192 \lambda+576 & 192 \lambda+96 \mu+222 & -96 \lambda-192 \mu-57 & 32 \lambda & 128 \mu-416 \lambda+240 \\
36-192 \lambda & 15-96 \lambda & 192 \lambda-30 & 0 & 24-128 \lambda \\
0 & -72 \mu-81 & 72 \mu+81 & -8 \mu-18 & 72 \mu+81 \\
192 \mu+540 & 24 \mu+171 & 9-120 \mu & -8 \mu-9 & 200 \mu+252
\end{array}\right) \text {, } \\
& \frac{1}{729}\left(\begin{array}{ccccc}
32 \mu \lambda-64 \mu-132 & -192 \lambda-33 & 192 \lambda+96 \mu+222 & 32 \lambda-9 & 224 \lambda+33 \\
64 \lambda-12 & 0 & 15-96 \lambda & 0 & 0 \\
-16 \mu-99 & 48 \mu-90 & -72 \mu-81 & -8 \mu-9 & 72-56 \mu \\
-80 \mu-171 & 48 \mu-90 & 24 \mu+171 & -8 \mu-18 & 81-56 \mu
\end{array}\right) .
\end{aligned}
$$

Similarly we obtain $\left\{\left.T_{\varepsilon}^{\boldsymbol{B}}\right|_{V_{0}^{\boldsymbol{B}}}: \varepsilon \in\{0,1,2\}\right\}$ given by (to be scaled by 1/243)

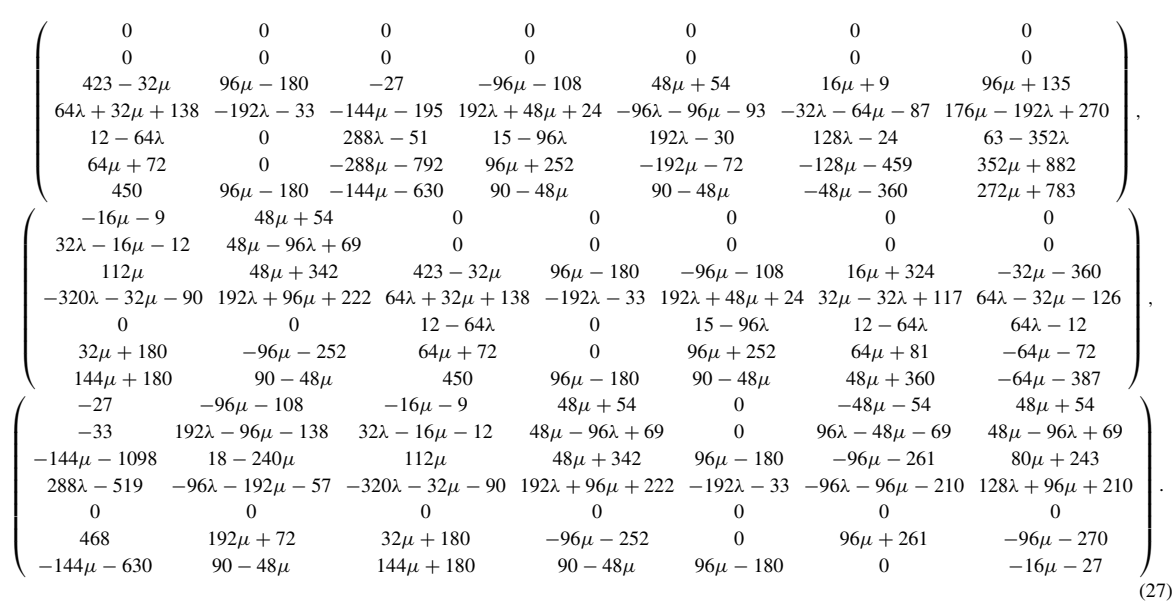


The mask $\boldsymbol{B}$ derived using the Taylor operator approach is given by (to be scaled by $1 / 243$ )

$$
\left.\begin{array}{lll}
\boldsymbol{B}(-5)=\left(\begin{array}{cc}
-16 \mu-9 & 32 \lambda-16 \mu-12 \\
48 \mu+54 & 48 \mu-96 \lambda+69
\end{array}\right), & \boldsymbol{B}(-4)=\left(\begin{array}{cc}
-32 \mu-45 & 64 \lambda-32 \mu-57 \\
0 & 0 \\
342-80 \mu & 160 \lambda-80 \mu+414 \\
16 \mu+747 & 819-16 \mu-128 \lambda \\
48 \mu-234 & 48 \mu-96 \lambda-282
\end{array}\right), & \boldsymbol{B}(-2)=\left(\begin{array}{cc}
48 \mu-234 & 192 \lambda+144 \mu-138
\end{array}\right), \\
\boldsymbol{B}(-3)=\left(\begin{array}{cc}
108-32 \mu-128 \lambda-96 \mu-327 \\
0 & 243
\end{array}\right), & \boldsymbol{B}(0)=\left(\begin{array}{cc}
-80 \mu-99-320 \lambda-240 \mu-387 \\
48 \mu+54 & 192 \lambda+144 \mu+150
\end{array}\right), \\
\boldsymbol{B}(-1)=\left(\begin{array}{cc}
32 \mu+45 & 160 \lambda+96 \mu+261 \\
0 & -96 \lambda-48 \\
0 & 0 \\
0 & 160 \lambda-27 \\
0 & 12-64 \lambda \\
0 & 15-96 \lambda
\end{array}\right), & \boldsymbol{B}(2)
\end{array}\right) .
$$

Last, we provide the computation of the entries (see (25)) of $\tilde{\boldsymbol{B}}_{+}^{*}(z)=$

$$
\begin{aligned}
& \left(\begin{array}{lll}
\tilde{b}_{00}(z) & \tilde{b}_{01}(z) & \tilde{b}_{02}(z) \\
\tilde{b}_{10}(z) & \tilde{b}_{11}(z) & \tilde{b}_{12}(z) \\
\tilde{b}_{20}(z) & \tilde{b}_{21}(z) & \tilde{b}_{22}(z)
\end{array}\right): \\
& \tilde{b}_{00}(z)=\frac{80 \mu z^{6}+99 z^{6}-32 \mu z^{5}-45 z^{5}+16 \mu z^{4}-126 z^{4}+112 \mu z^{3}+279 z^{3}-32 \mu z^{2}+108 z^{2}+16 \mu z+9 z+32 \mu+45}{81 z^{2}} \\
& \tilde{b}_{01}(z)=-\frac{320 \mu z^{6}+640 \lambda z^{6}+315 z^{6}-128 \mu z^{5}-256 \lambda z^{5}-51 z^{5}+64 \mu z^{4}+128 \lambda z^{4}-273 z^{4}}{324 z^{2}} \\
& -\frac{128 \mu z^{3}-384 l z^{3}+483 z^{3}+256 \lambda z^{2}+177 z^{2}-128 \lambda z+39 z-256 \lambda+48}{324 z^{2}} \\
& \tilde{b}_{02}(z)=\frac{z\left(160 \mu z^{3}+640 \lambda z^{3}+117 z^{3}-64 \mu z^{2}-256 \lambda z^{2}+39 z^{2}+32 \mu z+128 \lambda z-21 z+64 \mu+256 \lambda+42\right)}{324} \\
& \tilde{b}_{10}(z)=\frac{2(z-1)(z+1)\left(8 \mu z^{4}+9 z^{4}-8 \mu z^{3}-9 z^{3}+16 \mu z^{2}-30 z^{2}-8 \mu z-9 z+8 \mu+9\right)}{27 z^{2}} \\
& \tilde{b}_{11}(z)=-\frac{(z-1)\left(32 \mu z^{5}+64 \lambda z^{5}+35 z^{5}+36 z^{4}+32 \mu z^{3}+64 \lambda z^{3}-43 z^{3}-64 \lambda z^{2}-68 z^{2}-9 z-64 \lambda+10\right)}{54 z^{2}} \\
& \tilde{b}_{12}(z)=\frac{z\left(16 \mu z^{3}+64 \lambda z^{3}+17 z^{3}-16 \mu z^{2}-64 \lambda z^{2}+19 z^{2}+16 \mu z+64 \lambda z-z-16 \mu-64 \lambda-8\right)}{54} \\
& \tilde{b}_{20}(z)=0 \quad \tilde{b}_{21}(z)=-\frac{(z-1)(z+1)\left(z^{2}+z+1\right)^{2}}{6 z^{2}}, \quad \tilde{b}_{22}(z)=\frac{z(z+1)\left(z^{2}+z+1\right)}{6} .
\end{aligned}
$$

Acknowledgements The authors thank 'Vienna Scientific Cluster' (VSC) for providing computational resources. The authors thank the referees for their carefully reading of the paper and for the constructive comments and suggestions.

Funding Open access funding provided by Università degli Studi di Firenze within the CRUI-CARE Agreement. Costanza Conti received partial support from INdAM-GNCS. Maria Charina and Thomas Mejstrik were sponsored by the Austrian Science Foundation (FWF) grant P33352-N.

Open Access This article is licensed under a Creative Commons Attribution 4.0 International License, which permits use, sharing, adaptation, distribution and reproduction in any medium or format, as long as you give appropriate credit to the original author(s) and the source, provide a link to the Creative Commons licence, and indicate if changes were made. The images or other third party material in this article are included in the article's Creative Commons licence, unless indicated otherwise in a credit line to the material. If material is not included in the article's Creative Commons licence and your intended use is not permitted by statutory regulation or exceeds the permitted use, you will need to obtain permission directly from the copyright holder. To view a copy of this licence, visit http://creativecommons.org/licenses/by/4. $0 \%$ 


\section{References}

1. Cabrelli, C.A., Heil, C., Molter, U.M.: Self-similarity and multiwavelets in higher dimensions. Memoirs Amer. Math. Soc. 170(807) (2004)

2. Cavaretta, A.S., Dahmen, W., Micchelli, C.A.: Stationary subdivision. Memoirs Amer. Math. Soc. 93(453) (1991)

3. Charina, M.: Vector multivariate subdivision schemes: Comparison of spectral methods for their regularity analysis. Appl. Comp. Harm. Anal. 32, 86-108 (2012)

4. Charina, M., Conti, C., Guglielmi, N., Protasov, V.Y.: Limits of level and parameter dependent subdivision schemes: a matrix approach. Appl. Math. Comput. 271, 20-27 (2016)

5. Charina, M., Conti, C., Sauer, T.: Regularity of multivariate vector subdivision schemes. Numer. Alg. 39, 97-113 (2005)

6. Charina, M., Mejstrik, T.: Multiple multivariate subdivision schemes: Matrix and operator approaches. J. Comput. Appl. Math. 349, 279-291 (2019)

7. Chen, D.R., Jia, R.Q., Riemenschneider, S.D.: Convergence of vector subdivision schemes in Sobolev spaces. Appl. Comp. Harm. Anal. 12, 128-149 (2002)

8. Cohen, A., Dyn, N.: Nonstationary subdivision schemes and multiresolution analysis. SIAM J. Math. Anal. 27, 1745-1769 (1996)

9. Conti, C., Cotronei, M., Sauer, T.: Factorization of Hermite subdivision operators preserving exponentials and polynomials. Adv. Comput. Math. 42, 1055-1079 (2016)

10. Conti, C., Hüning, S.: An algebraic approach to polynomial reproduction of Hermite subdivision schemes. J. Comput. Appl. Math. 349, 302-315 (2019)

11. Conti, C., Merrien, J.L., Romani, L.: Dual Hermite subdivision schemes of de Rham-type. BIT Numer. Math. 54, 955-977 (2014)

12. Conti, C., Zimmermann, G.: Interpolatory Rank-1 vector subdivision schemes. CAGD 21, 341-351 (2004)

13. Dahmen, W., Micchelli, C.A.: Biorthogonal wavelet expansions. Const. Approx. 13, 293-328 (1997)

14. Daubechies, I., Lagarias, J.: Two-scale difference equations. II. Local regularity, infinite products of matrices and fractals. SIAM J. Math. Anal. 23, 1031-1079 (1992)

15. Dubuc, S., Merrien, J.L.: Hermite subdivision schemes and Taylor polynomials. Constr. Approx. 29, 219-245 (2009)

16. Dyn, N. In: Light, W. (ed.): Subdivision schemes in Computer-Aided Geometric Design, Advances in Numerical Analysis II Wavelets, Subdivision Algorithms and Radial Basis Functions, pp. 36-104. Clarendon Press, Oxford (1992)

17. Dyn, N., Levin, D.: Analysis of Hermite-interpolatory subdivision schemes. In: Dubuc, S., Deslauriers, G. (eds.) Spline Functions and the Theory of Wavelets, Amer. Math. Soc., Providence R. I., pp. 105-113 (1999)

18. Dyn, N., Moosmüller, C.: Increasing the smoothness of vector and Hermite subdivision schemes. IMA J. Numer. Anal. 39, 579-606 (2019)

19. Guglielmi, N., Protasov, V.Y.: Exact computation of joint spectral characteristics of matrices. Found. Comput. Math. 13, 37-97 (2013)

20. Guglielmi, N., Protasov, V.Y.u.: Invariant polytopes of sets of matrices with applications to regularity of wavelets and subdivisions. SIAM J. Matrix Anal. Appl. 37, 18-52 (2016)

21. Han, B., Overton, M., Yu, T.P.: Design of Hermite subdivision schemes aided by spectral radius optimization. SIAM J. Sci. Comput. 25, 643-656 (2003)

22. Hassan, M.F., Dodgson, N.A., Cohen, A., Merrien, J.L., Schumaker, L.L.: Ternary and three-point univariate subdivision schemes. In: Curve and Surface Fitting: Saint-Malo 2002, pp. 199-208. Nashboro Press (2003)

23. Hassan, M.F., Ivrissimtzis, I.P., Dodgson, N.A., Sabin, M.A.: An interpolating 4-point C2 ternary stationary subdivision scheme. Comput. Aided Geom. Des. 19, 1-18 (2002)

24. Jeong, B., Yoon, J.: Analysis of non-stationary Hermite subdivision schemes reproducing exponential polynomials. J. Comput. Appl. Math. 349, 452-469 (2019)

25. Jia, R.Q., Riemenschneider, S.D., Zhou, D.X.: Vector subdivision schemes and multiple wavelets. Math. Comp. 67, 1533-1563 (1998) 
26. Merrien, J.L.: A family of Hermite interpolants by bisection algorithms. Numer. Alg. 2, 187-200 (1992)

27. Merrien, J.L., Sauer, T.: From Hermite to stationary subdivision schemes in one or several variables. Adv. Comput. Math. 36, 547-579 (2012)

28. Merrien, J.L., Sauer, T.: Extended hermite subdivision schemes. J. Comput. Appl. Math. 317, 343-362 (2017)

29. Merrien, J.L., Sauer, T.: Generalized Taylor operators and polynomial chains for Hermite subdivision schemes. Numer. Math. 142, 167-203 (2019)

30. Mejstrik, T.: t-toolboxes, MATLAB Central File Exchange, mathworks.com/matlabcentral/ fileexchange/70449

31. Mejstrik, T.: Algorithm 1011: Improved invariant polytope algorithm and applications. ACM Trans. Math. Softw. 46(3), 26 (2020). Article 29. https://doi-org.uaccess.univie.ac.at/10.1145/3408891

32. Moosmüler, C., Hüning, S., Conti, C.: Stirling numbers and Gregory coefficients for the factorization of Hermite subdivision operators. IMA journal of Numerical Analysis, https://doi.org/10.1093/imanum/draa047, Published: 27 August 2020 (2020)

33. Rota, G.C., Strang, G.: A note on the joint spectral radius. Kon. Nederl. Acad. Wet. Proc. 63, 379-381 (1960)

Publisher's note Springer Nature remains neutral with regard to jurisdictional claims in published maps and institutional affiliations. 